\title{
ON THE MEDIANS OF GAMMA DISTRIBUTIONS AND AN EQUATION OF RAMANUJAN
}

\author{
K. P. CHOI
}

(Communicated by Wei Y. Loh)

\begin{abstract}
For $n \geq 0$, let $\lambda(n)$ denote the median of the $\Gamma(n+1,1)$ distribution. We prove that $n+\frac{2}{3}<\lambda(n) \leq \min \left(n+\log 2, n+\frac{2}{3}+(2 n+2)^{-1}\right)$. These bounds are sharp. There is an intimate relationship between $\lambda(n)$ and an equation of Ramanujan. Based on this relationship, we derive the asymptotic
\end{abstract} expansion of $\lambda(n)$ as follows:

$$
\lambda(n)=n+\frac{2}{3}+\frac{8}{405 n}-\frac{64}{5103 n^{2}}+\frac{2^{7} \cdot 23}{3^{9} \cdot 5^{2} n^{3}}+\cdots .
$$

Let median $\left(Z_{\mu}\right)$ denote the median of a Poisson random variable with mean $\mu$, where the median is defined to be the least integer $m$ such that $P\left(Z_{\mu} \leq m\right) \geq \frac{1}{2}$. We show that the bounds on $\lambda(n)$ imply

$$
\mu-\log 2 \leq \operatorname{median}\left(Z_{\mu}\right)<\mu+\frac{1}{3} .
$$

This proves a conjecture of Chen and Rubin. These inequalities are sharp.

\section{INTRODUCTION AND STATEMENT OF MAIN RESULTS}

For a continuous random variable $X$, the median is defined to be the least $x \in(-\infty, \infty)$ such that $P(X \leq x)=\frac{1}{2}$; and if $X$ is an integer-valued random variable, the median of $X$ is defined to be the least integer $m$ such that $P(X \leq$ $m) \geq \frac{1}{2}$. In either case, we denote the median of $X$ by median $(X)$. For $n \geq 0$, let $\lambda(n)$ denote the median of the $\Gamma(n+1,1)$ distribution, the Gamma distribution with parameters $n+1$ and 1 . In other words,

$$
\frac{1}{n !} \int_{0}^{\lambda(n)} t^{n} e^{-t} d t=\frac{1}{n !} \int_{\lambda(n)}^{\infty} t^{n} e^{-t} d t=\frac{1}{2}
$$

One of the main results in this paper is

Theorem 1. For $n \geq 0$,

$$
n+\frac{2}{3}<\lambda(n) \leq \min \left(n+\log 2, n+\frac{2}{3}+(2 n+2)^{-1}\right) .
$$

These bounds are sharp.

Received by the editors August 14, 1992.

1991 Mathematics Subject Classification. Primary 62E10, 41A58, 33B15.

Key words and phrases. Median, Gamma distribution, Poisson distribution, chi-square distribution, Poisson-Gamma relation, Ramanujan's equation. 
In this paper, we also consider the median of the Poisson distribution. Let $Z_{\mu}$ denote a Poisson random variable with mean $\mu$. Then it will be shown in the next section that Theorem 1 implies the following

Theorem 2. Let $\mu \in(0, \infty)$. Then

$$
\mu-\log 2 \leq \operatorname{median}\left(Z_{\mu}\right)<\mu+\frac{1}{3} .
$$

These bounds are best possible.

This proves Conjecture 1a of Chen and Rubin [2]. Based on a numerical study, Chen and Rubin were led to a stronger conjecture, which states that $\lambda(n)-n$ is decreasing in $n$. This still remains open. At the end of this section, we see that the coefficients of the first four terms of the asymptotic expansion of $\lambda(n-1)-(n-1)$ are positive. This strongly suggests that their conjecture is very likely to hold.

The work of this paper is also motivated by its connection with a well-known equation of Ramanujan. Ramanujan [7] and also his first letter to Hardy on January 16, 1913 contained the following statement: For $n \geq 1$, let $\theta(n)$ be defined as

$$
\frac{e^{n}}{2}=\sum_{k=0}^{n-1} \frac{n^{k}}{k !}+\theta(n) \frac{n^{n}}{n !} ;
$$

then $\frac{1}{3} \leq \theta(n) \leq \frac{1}{2}$. He also gave the first four terms of the asymptotic expansion of $\theta(n)$ :

$$
\theta(n)=\frac{1}{3}+\frac{4}{135 n}-\frac{8}{2835 n^{2}}-\frac{16}{8505 n^{3}}+O\left(\frac{1}{n^{4}}\right) .
$$

This problem has attracted a lot of attention. For example, Szegö [9] and Watson [10] proved that $\theta(n)$ lies between $\frac{1}{3}$ and $\frac{1}{2}$, whereas further coefficients of the asymptotic expansion have been found by Bowman, Shenton, and Szekeres [1] and Marsaglia [6]. Knuth considered the asymptotic expansion of $P\left(Z_{n} \leq n-1\right) / P\left(Z_{n}=n\right)$ from which he gave another derivation of the asymptotic expansion of $\theta(n)$, though with fewer terms (see Knuth [5, pp. $112-118])$. We find that there is an intimate relationship between $\lambda(n)$ and $\theta(n)$ (see the first paragraph of $\S 3$ ); namely,

$$
1-\theta(n)=\int_{n}^{\lambda(n)} t^{n-1} e^{-t} / n^{n-1} e^{-n} d t
$$

This will enable us to deduce bounds on $\theta(n)$ based on Theorem 1 and, conversely, asymptotic expansion of $\lambda(n)$ based on that of $\theta(n)$.

Theorem 3. With $\theta(n)$ defined as above and $n \geq 1$, we have

$$
\frac{1}{3}-\frac{73}{162 n}+\frac{19}{243 n^{2}}<\theta(n)<\frac{1}{3}+\frac{4}{81 n}-\frac{4}{243 n^{3}} .
$$

The upper bound in Theorem 3 is a decreasing function in $n$, and hence $\theta(n)<\frac{1}{3}+\frac{8}{243}=0.36625 \ldots$, which is an improvement of the upper bound $\frac{1}{2}$. 
Theorem 4. With $\lambda(n)$ defined as above, we have

$$
\lambda(n)=n+\frac{2}{3}+\frac{8}{405 n}-\frac{64}{5103 n^{2}}+\frac{2^{7} \cdot 23}{3^{9} \cdot 5^{2} n^{3}}+O\left(\frac{1}{n^{4}}\right)
$$

The first three terms of the asymptotic expansion can also be derived from Dinges [3, Proposition 3.6]. Our method here is different, and with more work many more terms can be derived. Doodson [4] considered

$$
\frac{\operatorname{median}\left(X_{n}\right)-\operatorname{mode}\left(X_{n}\right)}{\operatorname{mean}\left(X_{n}\right)-\operatorname{mode}\left(X_{n}\right)}=\operatorname{median}\left(X_{n}\right)-(n-1),
$$

where $X_{n}$ denotes a Gamma random variable with parameters $n$ and 1 . Using our notation and results, this ratio is

$$
\lambda(n-1)-(n-1)=\frac{2}{3}+\frac{8}{405 n}+\frac{144}{3^{6} \cdot 5 \cdot 7 n^{2}}+\frac{2^{3} \cdot 281}{3^{9} \cdot 5^{2} \cdot 7 n^{3}}+\cdots .
$$

\section{Proofs OF THEOREMS 1 AND 2}

Proof of Theorem 1. Let $X_{n+1}$ be $\Gamma(n+1,1)$ distributed. For $x \in\left[\frac{2}{3}, 1\right], n \geq$ 0 , let $a_{n}(x)=P\left(X_{n+1} \geq n+x\right)$ and $A_{n}(x)=(n+1) !\left[a_{n+1}(x)-a_{n}(x)\right]$. For $n \geq 0$ and $t>0$, let $\psi_{n}(t)=t^{n+1} e^{-t}$. Therefore,

$$
\begin{aligned}
A_{n}(x) & =\int_{n+x+1}^{\infty} t^{n+1} e^{-t} d t-(n+1) \int_{n+x}^{\infty} t^{n} e^{-t} d t \\
& =(n+x)^{n+1} e^{-(n+x)}-\int_{n+x}^{n+x+1} t^{n+1} e^{-t} d t \\
& =\int_{n+x}^{n+x+1}\left[\psi_{n}(n+x)-\psi_{n}(t)\right] d t .
\end{aligned}
$$

We shall show at the end of the proof that

$$
\begin{gathered}
A_{n}(\log 2)>0, \quad n \geq 2 ; \\
A_{n}\left(\frac{2}{3}+(2 n+2)^{-1}\right)>0, \quad n \geq 1 ; \\
A_{n}\left(\frac{2}{3}\right)<0, \quad n \geq 1 .
\end{gathered}
$$

By the Central Limit Theorem, we have $a_{n}(x) \longrightarrow \frac{1}{2}$ as $n \longrightarrow \infty$. From (9) we have $a_{n}\left(\frac{2}{3}\right)$ is a strictly decreasing sequence; therefore, $a_{n}\left(\frac{2}{3}\right)>\frac{1}{2}$. Hence, $\lambda(n)>n+\frac{2}{3}, \quad n \geq 1$. Direct computation shows that $\lambda(0)=\log 2>\frac{2}{3}$, establishing inequality (2) in the case $n=0$. The second inequality is proved in the same way. To prove (7)-(9), we write $A_{n}(x)=B_{n}(x)+E_{n}(x)$, where $B_{n}(x)$ is due to the Taylor expansion of $\psi_{n}(x)$ at $n+1$ up to the third derivative and 
with $E_{n}(x)$ the error term. It is not difficult to derive that

$$
B_{n}(x)=\left\{\frac{(3 x-2)}{6(n+1)}-\frac{6 x^{2}-8 x+3}{12(n+1)^{2}}\right\} \psi_{n}(n+1),
$$

and there exist $\xi \in[n+x, n+1]$ and $\xi(t)$ lying between $n+1$ and $t$ such that

$$
E_{n}(x)=\frac{(1-x)^{4}}{4 !} \psi_{n}^{(4)}(\xi)-\int_{n+x}^{n+x+1} \frac{(t-n-1)^{4}}{4 !} \psi_{n}^{(4)}(\xi(t)) d t
$$

Write $\psi_{n}^{(k)}(t)=w_{k, n}(t) \psi_{n}(t) / t^{k}$ and $u_{k, n}(s)=w_{k, n}(n+1+s)$ for $s \in$ $[-(1-x), x] \subseteq[-1,1]$. It is straightforward to verify that $u_{4, n}(s)=s^{4}+$ $(n+1)\left[3(n-1)-8 s-6 s^{2}\right]$, it is decreasing in $s$, and $u_{4, n}(s) \geq u_{4, n}(1)>0$ when $n \geq 6$. For $n \geq 6$, this implies that $\psi_{n}^{(4)}(\xi(t))>0$; hence, $E_{n}(x)$ is bounded above by

$$
\frac{(1-x)^{4} u_{4, n}(-1)}{24(n+x)^{4}} \psi_{n}(n+1) \text {. }
$$

Therefore, $A_{n}\left(\frac{2}{3}\right)<B_{n}\left(\frac{2}{3}\right)+\psi_{n}(n+1) /\left[648(n+1)^{2}\right]<0$, proving (9) for $n \geq 6$. Remaining cases can be verified by direct calculation.

Next, for $n \geq 6, E_{n}(x)$ is bounded below by

$$
\frac{-\left[x^{5}+(1-x)^{5}\right] u_{4, n}(-1)}{120(n+x)^{4}} \psi_{n}(n+1)
$$

Since the leading term of $A_{n}(\log 2)$ is $(3 \log 2-2) /[6(n+1)]$, it is apparent that $A_{n}(\log 2)>0$ for $n$ sufficiently large. Indeed, it can be verified to be positive for $n \geq 6$. Also, we have $B_{n}\left(\frac{2}{3}+\frac{1}{2(n+1)}\right)=\left(\frac{2}{9(n+1)^{2}}-\frac{1}{8(n+1)^{4}}\right) \psi_{n}(n+1)$ and $E_{n}\left(\frac{2}{3}+\frac{1}{2(n+1)}\right)>\frac{-3}{40(n+1)^{2}} \psi_{n}(n+1)$. Hence, $A_{n}\left(\frac{2}{3}+\frac{1}{2(n+1)}\right)>0$. Remaining cases can be verified by direct calculation. Since $\lambda(0)=\log 2$ and $\lambda(n)-n \rightarrow \frac{2}{3}$ as $n \rightarrow \infty$, the bounds are sharp. This completes the proof of Theorem 1 .

Remark 1. The lower bound in (2) and hence the upper bound in Theorem 2 were proved by an ingenious and yet elementary method in Chen and Rubin [2].

Proof of Theorem 2. Making use of the Poisson-Gamma relation, which states that for $n \geq 0, \mu>0$

$$
P\left(Z_{\mu} \leq n\right)=P\left(X_{n+1} \geq \mu\right)
$$

we see that $P\left(Z_{\lambda(n)} \leq n\right)=\frac{1}{2}$ and, for $\lambda(n)<\mu \leq \lambda(n+1), \operatorname{median}\left(Z_{\mu}\right)$ $=n+1$. Since $\lambda(n+1)-\lambda(n)>n+1+\frac{2}{3}-(n+\log 2)>0.97$, for any $\mu \in(\log 2, \infty)$, there exists $n \geq 0$ such that $\lambda(n)<\mu \leq \lambda(n+1)$. Therefore,

$$
\operatorname{median}\left(Z_{\mu}\right)-\mu=n+1-\mu \geq n+1-\lambda(n+1) \geq-\log 2
$$

and

$$
\operatorname{median}\left(Z_{\mu}\right)-\mu=n+1-\mu<n+1-\lambda(n)<\frac{1}{3} .
$$

For $\mu \in(0, \log 2], \operatorname{median}\left(Z_{\mu}\right)=0$, so $-\log 2 \leq \operatorname{median}\left(Z_{\mu}\right)$ with equality holds when $\mu=\log 2$. Let $\mu=\lambda(n)+\epsilon$, where $\epsilon$ is an arbitrarily small positive number. Then median $\left(Z_{\mu}\right)-\mu=n+1-\lambda(n)-\epsilon=\frac{1}{3}-\epsilon+O\left(n^{-1}\right)$. 
This shows that the second inequality is sharp, and this completes the proof of Theorem 2.

\section{Proofs of Theorems 3 ANd 4}

It is obvious that equation (4) is equivalent to

$$
P\left(Z_{n} \leq n\right)-[1-\theta(n)] P\left(Z_{n}=n\right)=\frac{1}{2} .
$$

Recalling the Poisson-Gamma relation (see equation (10)) and the definitions of $\psi_{n}$ and $\lambda(n)$, we obtain (5).

Proof of Theorem 3. Recall the definition of $\psi_{n}(t)$. Then

$$
1-\theta(n)<\int_{n}^{n+2 / 3+(2 n)^{-1}} \psi_{n-1}(t) / \psi_{n-1}(n) d t .
$$

Expanding $\psi_{n-1}(t)$ at $t=n$ up to the second derivative and estimating the error term as in the proof of Theorem 1 , we can show that $\psi_{n-1}(t) / \psi_{n-1}(n)$ is bounded above by

$$
\begin{gathered}
1-\frac{(t-n)^{2}}{2 n}+\frac{(t-n)^{3}}{6 n^{3}} \max _{0 \leq s \leq 2 / 3+(2 n)^{-1}}\left((2+3 s) n-s^{3}\right) \\
\quad \leq 1-\frac{(t-n)^{2}}{2 n}+\frac{(t-n)^{3}}{6 n^{3}}\left(4 n+\frac{3}{2}\right)
\end{gathered}
$$

from which we obtain the lower bound for $\theta(n)$. As shown in the proof of Theorem 1, for $n \geq 6, \psi_{n-1}^{(4)}(t)>0$ on $\left[n, n+\frac{2}{3}\right]$. Therefore, $\psi_{n-1}^{\prime \prime \prime}(\xi) \geq$ $\psi_{n-1}^{\prime \prime \prime}(n)$. It now follows that

$$
\frac{\psi_{n-1}(t)}{\psi_{n-1}(n)} \geq 1-\frac{(t-n)^{2}}{2 n}+\frac{(t-n)^{3}}{3 n^{2}} .
$$

For $n \geq 6$, this inequality and Theorem 1 imply

$$
1-\theta(n) \geq \int_{n}^{n+2 / 3} \frac{\psi_{n-1}(t)}{\psi_{n-1}(n)} d t \geq \frac{2}{3}-\frac{4}{81 n}+\frac{4}{243 n^{2}},
$$

and the upper bound for $\theta(n)$ follows. It can be verified that the upper bound still holds for $1 \leq n \leq 5$. This completes the proof of Theorem 3 .

Proof of Theorem 4. Using the Taylor expansion of $\psi_{n-1}$ at $t=n$, we have

$$
1-\theta(n)=\sum_{k=0}^{N-1} \frac{\psi_{n-1}^{(k)}(n)}{\psi_{n-1}(n)} \frac{(\lambda(n)-n)^{k+1}}{(k+1) !}+R_{N} .
$$

We estimate the error term $R_{N}$ as follows. Recall that

$$
\psi_{n-1}^{(N)}(t)=w_{N, n-1}(t) \psi_{n-1}(t) / t^{N} .
$$


It is not difficult to show by induction that $\left\|w_{N, n-1}\right\|=\max \left\{\left|w_{N, n-1}(t)\right|: n \leq\right.$ $t \leq n+\log 2\} \leq N ! n^{N / 2}$. Therefore,

$$
\begin{aligned}
\left|R_{N}\right| & =\left|\int_{n}^{\lambda(n)} \frac{(t-n)^{N}}{N !} \frac{\psi_{n-1}^{(N)}(\xi(t))}{\psi_{n-1}(n)} d t\right| \leq \frac{(\lambda(n)-n)^{N+1}}{(N+1) !} \frac{\left\|w_{N, n-1}\right\|}{n^{N}} \\
& \leq \frac{(\lambda(n)-n)^{N+1}}{(N+1) n^{N / 2}} \rightarrow 0 \text { as } N \rightarrow \infty .
\end{aligned}
$$

Let $a_{k}=\psi_{n-1}^{(k)}(n) / \psi_{n-1}(n)$ for $k \geq 0$. It is easy to verify that $a_{0}=1, a_{1}=0$, and, for $k \geq 2, a_{k}=-(k-1)\left[a_{k-1}+a_{k-2}\right] / n$. Then we obtain

$$
1-\theta(n)=\sum_{k=0}^{\infty} a_{k} \frac{(\lambda(n)-n)^{k+1}}{(k+1) !} .
$$

From this we can derive the asymptotic expansion of $\lambda(n)$. This finishes the proof.

\section{REMARKS}

It is not difficult to see that $\lambda$ median $(\Gamma(\alpha, \lambda))=\operatorname{median}(\Gamma(\alpha, 1))$. Therefore, we have the following corollary from Theorem 1 .

Corollary 5. For $n \geq 1$ and $\lambda>0$, we have

$$
\frac{-1}{3 \lambda} \leq \operatorname{median}(\Gamma(n, \lambda))-\operatorname{mean}(\Gamma(n, \lambda)) \leq \frac{-1+\log 2}{\lambda} .
$$

The bounds are best possible.

Using the fact that $\chi_{n}^{2}=\Gamma\left(\frac{n}{2}, \frac{1}{2}\right)$, we have

Corollary 6. For $m \geq 1$,

$$
-\frac{2}{3} \leq \operatorname{median}\left(\chi_{2 m}^{2}\right)-\operatorname{mean}\left(\chi_{2 m}^{2}\right) \leq 2(-1+\log 2) .
$$

The bounds are best possible.

\section{ACKNOWLEDGMENT}

I would like to thank the referee for many helpful suggestions which improved the paper.

\section{REFERENCES}

1. K. O. Bowman, L. R. Shenton, and G. Szekeres, A Poisson sum up to the mean and a Ramanujan Problem, J. Statist. Comput. Simulation 20 (1984), 167-173.

2. J. Chen and H. Rubin, Bounds for the difference between median and mean of Gamma and Poisson distributions, Statist. Probab. Lett. 4 (1986), 281-283.

3. H. Dinges, Special cases of second order Wiener Germ approximations, Probab. Theory Related Fields 83 (1989), 5-57.

4. A. T. Doodson, Relation of the mode, median and mean in frequency curves, Biometrika 11 (1971), 425-429.

5. D. E. Knuth, The art of computer programming, Vol. 1, 2nd ed., Addison-Wesley, Reading, MA, 1973. 
6. J. C. W. Marsaglia, The incomplete Gamma function and Ramanujan's rational approximation to $e^{x}$, J. Statist. Comput. Simulation 24 (1986), 163-168.

7. S. Ramanujan, J. Indian Math. Soc. 3 (1911), 151-152.

8. Collected Papers, Chelsea, New York, 1927.

9. G. Szegö, Über einige von S. Ramanujan gestelle Aufgaben, J. London Math. Soc. 3 (1928), 225-232.

10. G. N. Watson, Theorems stated by Ramanujan (V): Approximations connected with $e^{x}$, Proc. London Math. Soc. (2) 29 (1927), 293-308.

Department of Mathematics, National University of Singapore, Singapore 0511, RePUBLIC OF SINGAPORE

E-mail address: matckp@nusvm.bitnet 